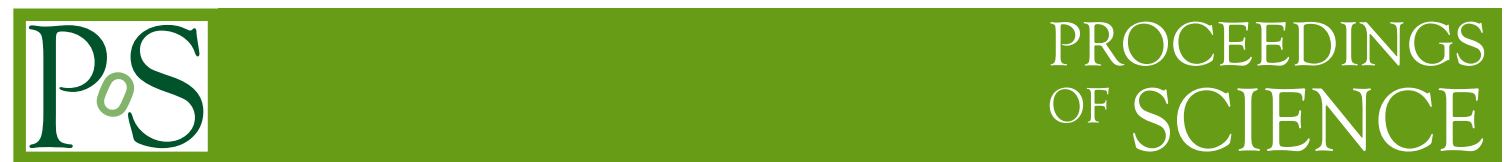

\title{
Double parton scattering studies in CMS
}

\author{
Rajat Gupta ${ }^{a, b}$ \\ a Panjab University, \\ Chandigarh, India \\ ${ }^{b}$ On behalf of the CMS and TOTEM Collaborations \\ E-mail: rajat.gupta@cern.ch
}

Recent results of the study of double parton scattering with the CMS experiment using states with a $\mathrm{Z}$ boson and jets, and with four jets will be presented.

\footnotetext{
*** The European Physical Society Conference on High Energy Physics (EPS-HEP2021), *** ***26-30 July $2021 * * *$

*** Online conference, jointly organized by Universität Hamburg and the research center DESY ***
} 


\section{Introduction}

Events in which two hard parton-parton interactions occur within a single proton-proton (pp) collision are referred to as double-parton scattering (DPS). Under the assumption of transverse and longitudinal factorization of the two single parton interactions, the DPS cross section can be written as:

$$
\sigma_{A B}^{D P S}=\frac{m}{2} \frac{\sigma_{A} \sigma_{B}}{\sigma_{e f f}}
$$

where $\mathrm{A}$ and $\mathrm{B}$ denote the single parton scattering (SPS) processes, and $\sigma_{A}$ and $\sigma_{A}$ their respective SPS cross sections. The factor ' $m$ ' is unity if processes A and B are the same, and $n=2$ if $\mathrm{A} \neq \mathrm{B}$. The parameter $\sigma_{e f f}$ is related to the extent of the parton distribution in the plane orthogonal to the direction of motion of the protons. In this document, recent CMS [1] measurements on the study of DPS using states with a $\mathrm{Z}$ boson and jets, and with four jets are presented.

\section{DPS study using inclusive four jets process}

Due to the complex structure of nucleons, it is possible to have more than one parton-parton interaction within the same proton-proton (pp) collision. DPS corresponds to events where two hard parton-parton interactions occur in single pp collisions. A study of inclusive four-jet production in pp collisions at a center-of-mass energy $(\sqrt{s})$ of $13 \mathrm{TeV}$ is presented [2]. Two phase space regions defined by selections on jet transverse momentum $\left(p_{T}\right)$ are used. In region I, the four leading jets within pseudorapidity $|\eta|<4.7$, are required to exceed $p_{T}$ thresholds of 35, 30, 25, and $20 \mathrm{GeV}$. Asymmetric thresholds have been chosen over symmetric ones because the latter tend to dampen the DPS contribution with respect to the single parton scattering (SPS) fraction. The $\Delta S$ (azimuthal angle between the hardest and the softest jet pair) distribution is obtained for region II, with $p_{T}$ thresholds of 50, 30, 30, and $30 \mathrm{GeV}$.

The $\Delta S$ distribution is less affected by different parton shower implementations. The DPS tune CDPSTP8S1-4j agrees very well with the shape, whereas all other models underestimate the data at low $\Delta S$, indicating a possible need for more DPS contribution.

The DPS contribution is extracted by means of a template fit to the data, using distributions for SPS obtained from Monte Carlo event generators and a DPS distribution constructed from inclusive single-jet events in data. Figure 1 shows the results for $\sigma_{\text {eff }}$ extracted with the models that are based on the recent $\mathrm{CP} 5$ and $\mathrm{CH} 3$ tunes and where the hard MPI have been removed. All results, except for the values obtained with the NLO $2 \rightarrow 2$ models, agree with the measurement performed by the ATLAS collaboration at a $\sqrt{s}$ of $7 \mathrm{TeV}$, where a $\sigma_{\text {eff }}$ equal to $14.9_{-1.0}^{+1.2}(\mathrm{stat})_{-3.8}^{+5.1}$ (syst) mb was found, while none agree with the value of $21.3_{-1.6}^{+1.2} \mathrm{mb}$ from the CMS measurement at a $\sqrt{s}$ of $7 \mathrm{TeV}$, which is more in line with the results obtained with some of the models based on older underlying event (UE) tunes.

\section{DPS study using inclusive $\mathrm{Z}+$ jets process}

A first measurement is performed to explore observables sensitive to the presence of DPS using the $\mathrm{Z}+$ jets process with the CMS detector at $\sqrt{s}=13 \mathrm{TeV}$, where the $\mathrm{Z}$ boson decays into two 
oppositely charged muons [3]. Jets are required to have a lower $p_{T}$ threshold of $20 \mathrm{GeV}$.

The production cross sections in the fiducial region are measured to be $158.5 \pm 0.3$ (stat) \pm 7.0 (syst) \pm 1.2 (theo) \pm 4.0 (lumi) pb for $Z+\geq 1$ jet events and $44.8 \pm 0.4$ (stat) \pm 3.7 (syst) \pm 0.5 (theo) \pm 1.1 (lumi) pb for $\mathrm{Z}+\geq 2$ jets events. The measured cross sections are described, within uncertainties, by various simulations except for the MG5_aMC + PYTHIA 8 (with DPS specific CDPSTP8S1-WJ tune). The cross section of the DPS-specific tune is predicted $10 \%$ higher than the measured cross section.

Figure 2 shows the differential cross section measurement as a function of $\Delta \phi$ between the $\mathrm{Z}$ boson and the leading jet. The Z+jets calculation of MG5_aMC + PYTHIA 8 without MPI is lower than the measurement by $50 \%$ at lower $\Delta \phi$ indicating sensitivity of this distribution to MPI. Different $\mathrm{MC}$ event generators describe, within uncertainties, the differential cross section as a function of $\Delta \phi$ except for the MG5_aMC + PYTHIA 8 predictions with the DPS-specific tune CDPSTP8S1-WJ, which shows a deviation up to 10-20\%, but correctly describes the shape of the observable (not shown in Figure). The presented results are a significant input to further improve the DPS-specific tunes and a global tune in combination with other soft QCD measurements in pp interactions at $\mathrm{TeV}$ scale.

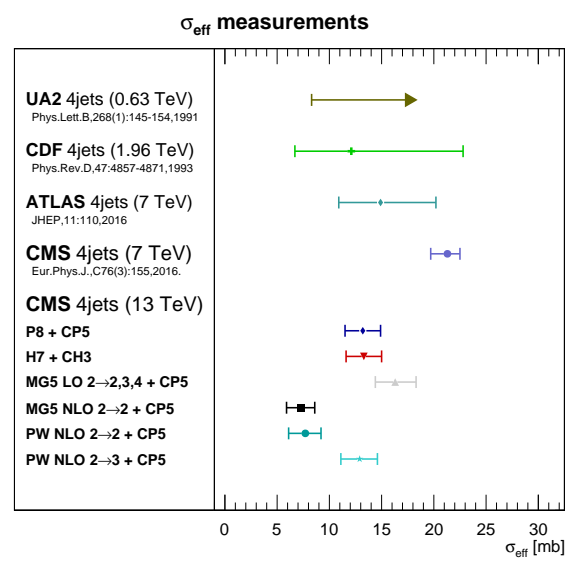

Figure 1: Comparison of the values for $\sigma_{\text {eff }}$ extracted from data. The results from four-jet measurements performed at lower $\sqrt{s}$ are shown alongside the newly extracted values [2].

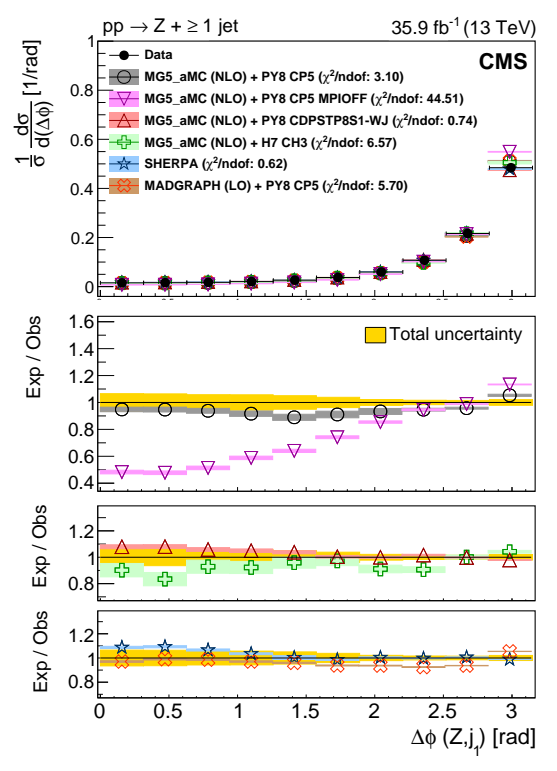

Figure 2: Differential cross sections as a functiona of $\Delta \phi$ between the $\mathrm{Z}$ boson and the leading jet for $Z+\geq 1$ jet events. In the bottom panels, the total uncertainty for data is indicated by the solid yellow band centred at 1. [3].

\section{Summary}

An overview of recent DPS measurements performed at CMS has been presented. The DPS measurements with 4 -jets and $\mathrm{Z}+$ jets processes demonstrate the need for further development of 
models in few areas. Apart from theoretical work and the work related to the development of MC tunes, new phase-space regions or variables sensitive to DPS effects are being identified and explored and their study is being pursued with more data from the LHC.

\section{References}

[1] CMS Collaboration, “The CMS Experiment at the CERN LHC," JINST 3 (2008) S08004

[2] CMS Collaboration, "Study of double-parton scattering in the inclusive production of four jets with low transverse momentum in proton-proton collisions at $\sqrt{s}=13 \mathrm{TeV}$," arXiv:2109.13822 (submitted to JHEP).

[3] CMS Collaboration, "Measurements of $\mathrm{Z}$ bosons plus jets using variables sensitive to double parton scattering in pp collisions at $13 \mathrm{TeV}$," arXiv:2105.14511 (accepted by JHEP). 